\title{
Nutrition and Gastrointestinal Microbiota, Microbial Derived Secondary Bile Acids, and Cardiovascular Disease
}

Jose Rodríguez-Morató, $\mathrm{PhD}^{1,2,3}$ and Nirupa R. Matthan, $\mathrm{PhD}^{4}$

${ }^{1}$ Integrative Pharmacology and Systems Neuroscience Group, IMIM, Hospital del Mar Medical Research Institute, Doctor Aiguader 88, 08003, Barcelona, Spain.

${ }^{2}$ Spanish Biomedical Research Centre in Physiopathology of Obesity and Nutrition (CIBEROBN), Instituto Salud Carlos III, 28029, Madrid, Spain

${ }^{3}$ Department of Experimental and Health Sciences, Universitat Pompeu Fabra (CEXSUPF), Dr. Aiguader 80, 08003, Barcelona, Spain

${ }^{4}$ Cardiovascular Nutrition Laboratory, Jean Mayer USDA Human Nutrition Research Center on Aging at Tufts University, Boston, MA

\section{Corresponding author:}

Nirupa R. Matthan, PhD.

Scientist I

Cardiovascular Nutrition Laboratory

Jean Mayer USDA Human Nutrition Research Center on Aging at Tufts University

711 Washington Street

Boston, MA 02111

Phone: (617) 556-3114

Fax: (617) 556-3103

nirupa.matthan@tufts.edu

\section{Keywords}

Diet, bile acids, gut microbiota, cardiometabolic risk factors, cardiovascular disease, metabolism. 


\section{ABSTRACT}

Purpose: The goal is to review the connection between gut microbiota and cardiovascular disease, with specific emphasis on bile acids, and the influence of diet in modulating this relationship.

Recent findings: Bile acids exert a much broader range of biological functions than initially recognized, including regulation of cardiovascular function through direct and indirect mechanisms. There is a bi-directional relationship between gut microbiota modulation of bile acid signaling properties, and their effects on gut microbiota composition. Evidence, primarily from rodent models and limited human trials, suggest that dietary modulation of the gut microbiome significantly impacts bile acid metabolism and subsequently host physiological response(s).

Summary: Available evidence suggests that the link between diet, gut microbiota and CVD risk, is potentially mediated via bile acid effects on diverse metabolic pathways. However, further studies are needed to confirm/expand and translate these findings in a clinical setting. 


\section{INTRODUCTION}

Although diet has long been known to contribute to the pathogenesis of cardiovascular disease (CVD), the interplay between dietary intake, gut microbiota and the host in modifying the risk of developing CVD is a recent area of investigation [1]. With regard to the association between diet and gut microbiota, both acute [2] and long-term dietary changes [3] have been shown to affect microbial communities. Evidence from animal models, as well as limited human research, suggest that the link between gut microbiota and CVD is potentially mediated via metabolites that are co-produced by both the host and gut microbiota during the metabolism of food and xenobiotics $[4,5]$.

Gut microbes can synthesize and transform an array of bioactive compounds, some of which remain in the gut, while others are absorbed into circulation where they can be co-metabolized by various tissues [6]. Some metabolites are only produced by gut bacteria, such as short chain fatty acids (SCFA) resulting from fermentation of dietary fiber, which have been shown to exert several beneficial CVD effects [5, 7]. Others reflect bacterial-specific modifications of host metabolites such as trimethylamine (TMA) derived from dietary choline, carnitine and phosphatidylcholine [6, 8], and secondary bile acids (SBA) derived from primary bile acids (PBA) [9]. Some co-metabolites are synthesized by host cells in response to bacterial signals $[9,10]$. Recently, both targeted and non-targeted [11] metabolomic studies have identified additional dietderived microbial metabolites such as uremic toxins (indoxyl sulphate and $p$-cresyl sulphate), derived from aromatic amino acids (AA: phenylalanine, tryptophan and tyrosine) during gut microbial and liver metabolism [12], and phenylacetylglutamine (PAG), a microbial product of AA fermentation, that are independent risk factors for CVD and mortality [13]. Thus, systematic analysis of microbial co-metabolites could provide novel insights into host-microbiota functional interactions. In this review, we focus on the role of one such family of metabolites, bile acids (BA), by summarizing their synthesis and metabolism, describing their biological functions especially in relation to CVD risk; highlighting the role of diet and other factors in modulating these relationships; and finally discussing the methodology for BA measurement.

\section{Synthesis and Metabolism}

BA are water-soluble, amphipatic primary end products of cholesterol metabolism [14]. The primary bile acids (PBA): cholic acid (CA) and chenodeoxycholic acid (CDCA) are synthesized in hepatocytes from cholesterol, via two different pathways. In adults, the classic or "neutral" pathway, accounts for $90-95 \%$ of BA synthesis, while the alternative or "acidic" pathway produces the remaining $5-10 \%$. Of note, the contribution of the latter pathway to the overall BA pool tends to be higher during childhood[15]. Cholesterol 7- $\alpha$ hydroxylase (CYP7A1) initiates the classic pathway and is the rate-limiting step, whereas sterol 27-hydroxylase (CYP27A1) initiates the alternative pathway [16]. After synthesis, BA are conjugated with glycine (major route) and taurine (minor route) (Figure 1), catalyzed by BA CoA synthetase (BACS) and BA CoA:amino acid Nacyltransferase (BAAT) to form bile salts, which are stored in the gallbladder. BA can also be sulfated at C-3 position by sulfotransferase 2A1, generating bis-conjugates [17], which are excreted in urine. Other minor reactions include ester glucuronidation, ethereal conjugation and $\mathrm{N}$-acetylglucosamination [18]. These conjugations lead to an increase in BA solubility and decrease in their toxicity [19]. BA synthesis is a tightly 
regulated process [20], with negative feedback inhibition mediated by the nuclear farnesoid $X$ receptor ( $F X R$ ), which is expressed in the liver, intestine and kidney [21]. While CA and CDCA are the major PBA in humans, other PBA have been described including hyocolic acid in pigs and muricholic acids in rodents [22].

After the ingestion of food, PBA are secreted via the bile duct into the duodenum, mediated by the hormones cholecystokinin and secretin[23]. The majority of BA (95\%) are reabsorbed from the terminal ileum and colon and delivered back to the liver via the portal vein in a process known as enterohepatic circulation, with only a minor amount $(\sim 5 \%)$ excreted in feces [17]. Typically, BA undergo between 4 to 10 enterohepatic cycles per day $[14,24]$. This process is an important physiological mechanism for recycling $B A$, nutrient absorption, and regulation of whole-body lipid metabolism[25]. In the intestine, the glycine and taurine moieties of PBA are deconjugated, catalyzed by bile salt hydrolase (BSH), and mediated by a broad spectrum of aerobic and anaerobic bacteria (Gram-positive Bifidobacterium, Lactobacillus, Clostridium and Enterococcus, and Gram-negative Bacteroides) [26]. Then, bacterial hydroxysteroid dehydrogenase $(\mathrm{HSDH})$ enzymes, remove a hydroxyl group at the 7a position and convert PBA: CA and CDCA into secondary bile acids (SBA): lithocholic acid (LCA) and deoxycholic acid (DCA), respectively (Figure 1). This dehydroxylation reaction is mediated by a limited number of bacteria belonging to Bacteroides, Clostridium, Eubacterium, Lactobacillus and Escherichia genera [27]. The SBA are absorbed from the colon and returned to the liver, where similar to the PBA, they can be conjugated and enter the enterohepatic pathway. Another bacterial transformation of BA is epimerization, mediated by a limited number of strict anaerobic bacteria (Actinobacteria, Proteobacteria, Firmicutes and Bacteroidetes) [26]. This mainly occurs in the large intestine [17, 24] and results in the production of the SBA, ursodeoxycholic acid (UDCA), which is present in low amounts in humans. These and other reactions (amidation, oxidation/reduction, esterification and desulfatation) [24] result in the production of numerous BA, the exact number in humans is still unclear. Methods that quantify BA generally monitor from 11 to 32 different BA, although a recent method detected 145 potential BA in human urine (55 BA) and blood (66 BA), respectively[28].

\section{Biological actions}

BA exert multiple physiological functions, which can be classified into three broad categories: (i) traditional, (ii) hormone-like, and (iii) antibacterial. For more than 50 years, BA were known for their important role in lipid metabolism by acting as intestinal detergents solubilizing dietary lipids/fat-soluble vitamins and promoting their absorption [14]. The conversion of cholesterol into BA and their biliary excretion, are the two main physiological mechanisms to remove cholesterol from the body [22]. Interestingly, in 1999, BA were documented to act as hormones [29], with the ability to modulate carbohydrate, lipid and cholesterol metabolism, as well as energy homeostasis [30, 31].

More recently, it has been documented that BA act as signaling molecules via two independent pathways: FXR (delayed signaling) [32] and G-protein coupled membrane receptor: the Takeda-G-protein-receptor 5 (TGR5) (rapid signaling) [15, 30]. BA have different affinities towards these receptors, with $C D C A>D C A>L C A>C A$ for FXR activation and $\mathrm{LCA}>\mathrm{DCA}>\mathrm{CDCA}>\mathrm{CA}$ for TGR5 activation. Thus TGR5 tends to be 
preferentially activated by SBA [26], although discrepancies between in vitro and in vivo affinities have been reported [33]. BA exert multiple biological functions via these receptors. Through FXR, BA can regulate their homeostasis, as well as lipogenesis, gluconeogenesis, tumor suppression, and intestinal barrier function [31]. Through TGR5, BA can regulate glucose homeostasis, energy expenditure, gallbladder relaxation and anti-inflammatory responses [31]. Additionally, BA are ligands of muscarinic receptors [34], sphingosine-1-phosphate receptor 2 [24], as well as nuclear receptors: pregnane $X$ receptor, vitamin $\mathrm{D}$ receptor, constitutive androstane receptor, and the liver $X$ receptor $\alpha[16,31]$.

Through their antibacterial properties, BA regulate gut microbiota composition and are important mediators of gut innate defense by providing protection against invasive organisms [35]. BA also maintain the sterility of the biliary tract [15], gut barrier integrity and modulate the immune system [36]. These effects can be either direct, by disrupting bacterial membranes and leaking cellular contents [35] or indirect, via inhibition of bacterial overgrowth and mucosal injury through FXR [30]. Of note, BA can become cytotoxic at high concentrations and excessive accumulation can lead to oxidative stress, apoptosis and liver damage [37].

\section{Relationship between bile acids and cardiovascular disease}

Observational studies have documented an association between plasma or fecal BA concentrations and CVD risk [38]. In a case-control study, elevated plasma concentrations of LCA, a SBA, correlated with a higher risk of CHD [39]. Similarly, individuals with coronary artery disease $(C A D)$ have been shown to have a reduced excretion of SBA (DCA and LCA) [40], and lower rates of BA synthesis and/or inefficient elimination of cholesterol [41]. A recent study involving 7,438 individuals confirmed earlier observations that patients with CAD had higher fasting serum total BA than individuals without CAD [42]. A retrospective study also found that lower SBA excretion was an independent risk factor for stroke and mortality[38]. Additionally, human clinical studies have documented associations between BA and intermediate CVD risk factors including regulation of lipid profile [43], glucose, and insulin resistance [44].

The causal role of BA in preventing atherosclerosis development has been studied mainly using tissue culture models [34], various knockout mouse models, and indirectly through use of agonists and antagonists of FXR and TGR5 receptors. While some animal studies have shown that PBAs through FXR activation can mitigate atherosclerosis development [45], improve lipid profiles and influence vascular tension [46], it has also been observed that FXR deficiency reduced atherosclerosis in male Ldlr-/- mice [47]. The activation of TGR5 by a semisynthetic BA has also been shown to protect against the development of atherosclerosis in animal models by inducing cAMP signaling and subsequent NF-kB inhibition, which decreased the production of proinflammatory cytokines [48]. Since BA receptors are expressed in cardiovascular tissue, they can interact with TGR5 receptors in the heart and systemic circulation, which has been suggested to reduce macrophage inflammation and lipid loading [48]. Interestingly, it has been suggested that the loss of one receptor (FXR or TGR5) could be compensated by the presence of the other [26]. Additionally, the simultaneous activation of FXR and TGR5 using a dual agonist (INT-767) has been documented to 
reverse obesity and atherosclerosis [49], whereas the dual deficiency of FXR and TGR5 exacerbated the development of atherosclerosis [50]. In addition to these receptordependent effects, BAs also exert receptor-independent effects on cardiovascular function via large conductance calcium-activated potassium channels that regulate arterial tone [34]. Through these pathways, BA can induce vasodilation (short-term effects) and can modulate the transcription of vasoactive molecules (long-term effects) [34]. Other postulated mechanisms include an enhancement of nitric oxide signaling, activation of muscarinic receptors and calcium-dependent potassium channels [45].

Conversely, SBA have also been implicated in atherosclerosis development by modulating $\mathrm{BSH}$ activity of specific gut microbiota, which as previously described, hydrolyze glycine- and taurine-conjugates to liberate free BA. These cytotoxic SBA have been shown to increase cholesterol levels, foam cell formation, and the size of the atherosclerotic plaque [51]. Taken together, in vitro and in vivo studies [46, 52] seem to suggest a cardiotoxic effect of hydrophobic SBAs (LCA, DCA) and cardioprotective effect of hydrophilic BAs (CA, CDCA), thereby highlighting the importance of BA chemical diversity as well as maintaining BA pool homeostasis in CVD development.

\section{Pharmacological modulation of bile acid metabolism and receptors}

Given the important biological role of BA, some pharmacological approaches target their metabolism and/or receptors in order to provide beneficial health effects. Most common are the BA sequestrants (resins) which are commercially available drugs (e.g. colestipol, colestyramine, and colesevelam) that bind to BA in the intestine and prevent their reabsorption. This leads to decreased enterohepatic recirculation of BA which is compensated with higher BA synthesis from cholesterol, thereby lowering circulating cholesterol concentrations [53]. Similarly, exogenously administered UDCA has been shown to decrease cholesterol absorption, increase BA biosynthesis, and decrease biliary cholesterol secretion [54]. The discovery that BA bind to FXR and TGR5 receptors has opened the possibility to target these receptors and modulate their corresponding signaling cascade. These include obeticholic acid (6a-ethyl derivative of CDCA), a semisynthetic BA and a potent FXR agonist [55], the INT-767 dual FXR/TGR5 agonist, the semi-synthetic BA 24-norursodeoxycholic acid and 12monoketocholic acid, all of which have shown to exert positive effects on metabolic disorders, as reviewed elsewhere[56].

\section{Factors that affect the relationship between bile acid and cardiovascular disease}

Diet: There is a large and growing body of evidence on the influence of specific diet components as well as dietary patterns on the human gut microbiome and subsequent CVD risk[57]. However, the involvement of gut-derived metabolites as potential mediators in this process is still an emerging area of research. To date, the most extensively studied metabolites implicated in CVD include TMA, its derivative trimethylamine $\mathrm{N}$-oxide (TMAO) [8], and SCFA [58]. Limited information is available regarding BA metabolism in response to dietary interventions. These studies have been discussed below and are summarized in Table 1.

Dietary fat: High dietary fat intake is known to increase PBA release into the small intestine and stimulating SBA synthesis, mediated via various bacteria, including 
Lactobacillus, Bifidobacterium and Bacteroides [59]. Interestingly, kinetic studies using stable isotopes indicate that both extremely low-fat and high-fat diets resulted in decreased turnover and synthesis of PBA, and consequently impaired removal of cholesterol from the body. This suggests that different mechanisms underlie the similar metabolic effects of the low-fat and high-fat diets [60]. Some studies have found differential physiologic effects of individual fatty acids based on food source [61,62]. Devkota et al. [63] showed that consumption of a diet high in SFA (milk fat) or n-6 PUFA (safflower oil) similarly increased Bacteroidetes and decreased Firmicutes abundances in genetically pre-disposed IL-10 $0^{-/}$mouse model for spontaneous colitis. However, the milk fat diet induced shifts in hepatic conjugation of BA, from glycocholic (GC) to taurocholic (TC) acid, and resulted in abundance of Bilophila wadsworthia, a biletolerant microbe. In a randomized cross-over clinical trial, Meng et al. [64] showed that consumption of a diet enriched in stearic acid (a longer chain SFA) resulted in lower fecal LA and total SBA concentrations than a MUFA diet enriched in oleic acid. Additionally, LA concentrations were positively correlated with fasting LDL-cholesterol concentrations, while total SBA, LA and DCA were negatively correlated with fasting HDL cholesterol concentrations [64]. The authors hypothesized that the hypocholesterolemic effect of stearic acid may be mediated by inhibition of intestinal hydrophobic SBA synthesis [64]. Together, available data suggest that dietary fat quantity (low fat versus high fat) as well as quality (fatty acid classes/individual fatty acids) can influence hepatic synthesis of PBA, and induce compositional changes in the gut microbiota, thus directly and indirectly altering SBA metabolism and CVD risk.

Dietary protein: Protein intake also has a significant impact on gut health[65]. Consumption of a high protein diet has been shown to increase circulating BA concentrations, and was associated with improvement in several metabolic markers in human and rodent models [66-68]. In a short-term human intervention study, the combination of a high fat, high protein diet significantly increased circulating BA concentrations compared to consumption of a high-fat diet alone [68].

With regard to protein type, compared to animal-based protein [57], plant-based protein intake has been associated with decreases in Bacteroides and increases in Bifidobacterium and Lactobacillus, all of which have been linked to favorable health outcomes [57, 69, 70]. Research using animal models suggests that this beneficial effect may be mediated via alterations in BA metabolism. Arellano-Martínez et al. documented that compared to casein, soy protein isolate lowered serum cholesterol concentration by increasing PBA synthesis via the repression of fibroblast growth factor 15 (FGF15) and small heterodimer partner (SHP), which in turn increased SBA excretion to prevent cholesterol overload in the enterocytes by increasing reverse cholesterol transport [67]. Another suggested mechanism relates to a microbiota-driven increase in BA transformation and increase in glucagon-like peptide-1 (GLP-1) secretion [71]. Similarly, dietary pea protein [72] has been shown to stimulate formation and excretion of SBA in rats, reducing hepatic cholesterol concentration and subsequent secretion of very low-density lipoprotein cholesterol (VLDL). Increased gene expression of sterol regulatory element-binding protein-2 (SREBP-2), 3-hydroxy-3-methyl-glutarylcoenzyme A reductase, and LDL receptor were also observed which suggest a compensatory mechanism to account for the shunting of cholesterol into the BA 
synthesis pathway [72]. Weichert et al. studied the effects of isoenergetic increases in the dietary protein and cereal-fiber content of the diet and observed markedly increased serum BA concentrations in obese, but not in overweight participants [73]. Possible mechanisms explaining this effect could be related to compensatory increases of the BA pool or defective transport. Interestingly, among the various protein sources in this study, only higher milk protein intake was a significant dietary predictor for changes in BA. These studies suggest that both protein source (animal versus plant based) as well as the amino acid composition of the protein source may be important regulators of BA metabolism.

Dietary carbohydrate: Several clinical and animal studies have documented that dietary carbohydrate intake can modulate gut microbiota and CVD risk [74], via SCFA production, as reviewed in detail elsewhere [58]. However, alterations in BA metabolism have also been noted. Specifically, the role of soluble and insoluble dietary fiber in binding BA is well documented in several studies [75]. Using a porcine model, Gunness et al. documented that oat beta-glucan was associated with modulation of the BA profile, reducing circulating levels as well as the ability of BA to diffuse across the terminal ileum [76]. In a recent randomized cross-over clinical study, the consumption of a diet rich in whole-grains, legumes, vegetables, and fruits, significantly increased the concentrations of taurolithocholic acid (TLCA), glycocholic acid (GCA), and taurocholic acid (TCA), compared to a refined grain diet (high glycemic load). Several of the BA

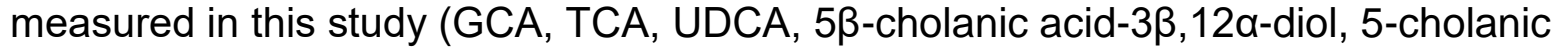
acid-3 $\beta$-ol, and glycodeoxycholic acid [GDCA]) were significantly positively associated with HOMA-IR [77]. Interestingly, sex and BMI specific differences in BA profiles were also observed. Since BA serve as ligands for FXR and TGR5, this may explain the beneficial effects on glucose homeostasis. Whole-grain foods have also been reported to reduce plasma LDL cholesterol concentrations with a concurrent increase in the relative abundance of fecal Bifidobacterium and Lactobacillus, which contribute to SCFA production and BA deconjugation [78]. The resulting increase in BA excretion is thought to contribute to an upregulation of hepatic LDL receptors and subsequent uptake of LDL cholesterol from the circulation [79]. Taken together, postulated mechanisms for the beneficial effect of whole grain/high fiber diets on lipid and glucose metabolism has been attributed, in part, to the ability of fiber to bind cholesterol, impeding cholesterol absorption and BA reabsorption, and modulating FXR and TGR5 receptors.

Limited work has evaluated the effect of mono-and disaccharides on BA metabolism. A small human study examined post-prandial plasma BA response to isocaloric beverages consisting primarily of carbohydrate (as glucose), protein or lipid [80]. The high-lipid beverage increased total plasma BA, whereas the high-carbohydrate beverage had little effect. Using a mouse model, sucrose intake was shown to reduce BA synthesis the hepatic mRNA expression of Cyp7a1. However, subsequent work indicated that it was not sugar per se in the high-sucrose diet that reduced BA synthesis, but rather the reduced content of fiber or fat [81].

Individual Foods/Nutrients: Several animal and in vitro studies have described that polyphenols (e.g. puerarin, resveratrol, and quercetin) can increase BA excretion, with corresponding reductions in total and LDL cholesterol, which may occur through FXR, 
NF-KB, and ERK signaling [82]. More recently, Rodríguez-Morató et al. have documented that cranberries, fed within the context of an animal based diet (rich in meats, dairy products, and simple sugars plus $30 \mathrm{~g} /$ day freeze-dried whole cranberry powder) relative to a control diet (animal-based diet plus $30 \mathrm{~g} /$ day placebo powder), had acute and profound effects on gut bacterial profiles that were potentially modulated by bacterially derived SBA (DCA), SCFA (acetate and butyrate), and phenolic acids[83].

Holscher et al. have documented that walnut consumption affected the composition and function of the human gut microbiota, increasing the relative abundances of butyrateproducing species, and reducing microbially derived, proinflammatory SBA (DCA and LA) and LDL cholesterol. Interestingly, abundance of Dorea and Roseburia were related to LA concentration, and a trend for a reduction in predicted bacterial SBA biosynthesis gene abundances was observed in the walnut compared with the control group. The authors speculated that the associations between the gut microbiota and cardiometabolic health may be related to microbial metabolism of BA and subsequent signally through FXR [84]. Of note, the observed enrichment of Roseburia following the walnut diet is a similar finding to Tindall and colleagues [85], who also reported that a diet enriched in whole walnuts or a diet with a matching fatty acid profile to the walnut diet, led to significant enrichment in Roseburia, Eubacteria eligens, and Butyricicoccus relative to a diet matched for SFA but with oleic acid in place of alpha linolenic acid (ALA). While BAs were not measured in this study, these results provide supporting evidence, albeit indirectly, that replacing SFAs with walnuts or a walnut fatty acid profile match may beneficially affect the gut environment through increasing bacteria that are inversely associated with SBA production.

Recent investigations show that choline and carnitine, originating from dietary fat and meat, are metabolized by the gut microbiota to TMA, which in turn can be converted to TMAO in the liver [86], and increase CVD risk and atherosclerosis development[86, 87]. Interesting, feeding apoE ${ }^{-/-}$mice TMAO has been shown to significantly increase serum BA profiles (tauromuricholic acid, DCA, CA), and downregulate the classical BA synthesis pathway, potentially mediated by activation of small heterodimer partner (SHP) and FXR, thus partially explaining the mechanisms by which TMAO contributes to CVD pathogenesis [88].

Probiotics are live microorganisms that are sold as food ingredients, including dairy products and over-the-counter capsules and powders [89]. Many of the widely consumed probiotics strains have been shown to alter BSH activity and the BA pool, with potential hypocholesterolemic properties [89, 90]. Animal work has shown that a decrease in BSH activity is associated with weight gain, with resultant effects on FXR signaling which can influence host pathways involved in lipid metabolism, peripheral circadian rhythm, gut barrier function, and immune homeostasis [91]. Also using a mouse model, the probiotic mixture VSL\#3 (containing BSH active probiotic strains) significantly increased fecal BA deconjugation and excretion, as well as hepatic BA synthesis [92]. In contrast, human clinical studies investigated the hypo-cholesterolemic effect of probiotics have not been conclusive [89]. 
Dietary patterns: Western diets characterized by high intakes of SFA, refined grains, and low intakes of dietary fiber, are correlated with higher CVD risk and higher colonic concentrations of SBA [93]. In contrast, vegan/vegetarian (low in SFA) and Mediterranean diets (rich in unsaturated fat), both of which are also high in fiber and phytochemicals, are related to lower fecal BA concentrations [94]. Interesting serum CA, CDCA, and glycine-conjugated BA concentrations are higher in vegans compared to omnivores and this appears to be due to increased intestinal reabsorption rather than increased hepatic synthesis [70, 77, 93]. There are several other popular diets that are being advocated for gut health such as strict vegan, raw food, gluten-free, and low FODMAP (fermentable oligosaccharides, disaccharides, monosaccharides, and polyols) diets. These diets tend to be restrictive, and their effects on BA metabolism and CVD risk factors are unknown.

Other factors: Differences in BA profiles between males and females have been reported in animal models, and have been implicated in the sex differences observed in various metabolic diseases [95, 96]. Drugs such as fibrates [37], statins [97], and antibiotics including ampicillin [98] and vancomycin [95] have been shown to modulate BA metabolism in mice and humans. Ethnic differences in BA have been reported in a limited number of studies but results are inconclusive [42]. More recently, bariatric surgical procedures to reduce body weight in individuals with obesity, have been shown to alter BA metabolism [99]. Also, circadian oscillations and inter-individual variations have also been reported in BA concentrations[100]. Thus, these additional factors must be considered during sample collection for measurement of BA, as well as potential confounders during data analyses.

\section{Measurement of bile acids}

The quantification of BA is challenging due to their complex chemical structure, differences in polarity, location of the hydroxyl groups (C3, $C 7$ and/or C12), the numerous bacterial-derived compounds, and the fact that some $\mathrm{BA}$ are isomers or stereoisomers [101]. Additional challenges include different BA profiles and concentrations depending on the matrix (plasma, bile, liver, urine or stool), as well as the species [102]. The choice of biological specimen to be collected should be dictated by the goals of the research study, as each matrix is differentially enriched in either PBAs, SBAs and/or their conjugates and metabolites. For instance, stool would be the matrix of choice to study microbially-derived SBAs; serum/plasma for circulating PBA and SBA especially glycol- and tauro-conjugates; and urine for phase II polar BA metabolites (e.g. sulfates, glucuronides). When studying specific diseases, additional specimens (liver, brain, or bile), if accessible, should be considered. Traditionally, BA were measured using thin layer chromatography and gas chromatography (GC) techniques. Additional methods include enzyme-linked immunosorbent assays (ELISA) and Nuclear Magnetic Resonance (NMR), which are limited by their low specificity and detection limit. More recent methods are based on liquid chromatography coupled to tandem mass spectrometry (LC-MS/MS) $[28,101]$, which overcome some of the GC limitations such as complex sample preparation steps, and have higher sensitivity and specificity [103]. Sample preparation strategies for BA measurement include either liquid-liquid extraction, solid-phase extraction or protein precipitation. Most LC-MS/MS 
methods are based on reverse phase chromatography, negative electrospray ionization and detection by multiple reaction monitoring [101]. BA conjugates, unlike unconjugated BA, generate characteristic fragment ions, so are easier to monitor. Several methods use pseudo-transitions and optimize chromatographic separation to accurately quantify unconjugated BA [104]. Some recent methods include simultaneous detection of free and conjugated BAs along with oxysterols (precursors of BA) [105]. A detailed review of current BA analytical methods has been conducted by Dutta et al. [106].

\section{CONCLUSION}

It is now recognized that BA exert a much broader range of biological functions than initially recognized, including acting as potent signaling molecules. Gut microbiota composition significantly influences BA metabolism, thus factors that modify the microbiome, especially diet (macronutrients and fiber), significantly alter the signaling properties of BA, with subsequent impact on host metabolism. Some recent studies have focused on individual nutrients/foods and have documented either favorable (whole grains, walnuts, cranberries, probiotics), or unfavorable (SFA, refined grains, TMAO) effects on BA metabolism and thus CVD risk. Among the dietary patterns studied, vegetarian and Mediterranean diets appear to have beneficial effects on BA metabolism compared to western-type diets. Thus, available evidence suggests that the link between diet, gut microbiota and CVD risk, is potentially mediated via BA effects on diverse metabolic pathways. However, it must be noted that the majority of these data are from animal/in vitro models, and observational studies, with limited human clinical data. Many of the human gut microbiome studies do not provide or collect information about BA concentrations in fecal or plasma samples in response to dietary interventions. Part of this might be due to methodological limitations but newer approaches are available to accurately quantify BA in various bio-specimens. Further studies are warranted to confirm and expand our understanding of the role of BA in mediating the relationship between gut microbiota and CVD risk, and to assess the translational nature of the findings in a clinical setting. 
Funding: This work was supported by the US Department of Agriculture, under agreement no. 58-1950-4-401. Any opinions, findings, conclusions, or recommendations expressed in this publication are those of authors, and do not necessarily reflect the views of the US Department of Agriculture, the National Institutes of Health. JRM acknowledges funding from European Union's Horizon 2020 research and innovation program under the Marie Skłodowska-Curie grant agreement No 712949 (TECNIOspring PLUS) and from ACCIO.

Conflicts of interest: None of the authors have any conflicts of interest related to this article.

Human and Animal Rights: All reported studies/experiments with human or animal subjects performed by the authors have been previously published and complied with all applicable ethical standards (including the Helsinki declaration and its amendments, institutional/national research committee standards, and international/national/ institutional guidelines). 


\section{Figures}

Figure 1

Schematic representation of bile acid synthesis and metabolism 
Table 1. Summary of recent studies evaluating the impact of dietary factors on bile acid metabolism and cardiovascular disease risk. ${ }^{1}$

\begin{tabular}{|c|c|c|c|}
\hline \multirow{2}{*}{$\begin{array}{l}\text { COMPONENT } \\
\text { Macronutrients }\end{array}$} & Model [reference] & Results and/or Suggested Mechanism & Metabolic Effect \\
\hline & & & \\
\hline \multirow[t]{5}{*}{ Dietary Fat } & $\begin{array}{l}\text { Rodent (rats) } \\
\text { [59] }\end{array}$ & $\begin{array}{l}\text { HF intake increased PBA release into the small intestine and stimulated } \\
\text { SBA synthesis, mediated via various gut bacteria. }\end{array}$ & Unfavorable \\
\hline & Human[60] & $\begin{array}{l}\text { Extremely HF or LF diets decreased turnover and synthesis of PBA, } \\
\text { relative to a moderate fat diet. }\end{array}$ & Unfavorable \\
\hline & $\begin{array}{l}\text { Rodent (mice IL10 } / \text { ) } \\
\text { [63] }\end{array}$ & $\begin{array}{l}\text { High SFA versus a PUFA n- } 6 \text { diets shifted hepatic conjugation of BA from } \\
\text { GCA to TCA and increased abundance of bile tolerant microbes. }\end{array}$ & Unfavorable \\
\hline & $\begin{array}{l}\text { Rodents (rat) } \\
\text { [107] }\end{array}$ & $\begin{array}{l}\text { Diet enriched in menhaden oil increased fecal BA excretion compared to } \\
\text { soybean oil and lard diets. FA excretion was positively correlated with } \\
\text { relative abundance of Firmicutes, and negatively correlated with relative } \\
\text { abundance of Bacteroidetes. }\end{array}$ & $\begin{array}{l}\text { Mixed depending } \\
\text { on fat source }\end{array}$ \\
\hline & $\begin{array}{l}\text { Human } \\
{[64]}\end{array}$ & $\begin{array}{l}\text { Stearic acid enriched diet lowered fecal LA and total SBA concentrations } \\
\text { compared to palmitic or oleic acid diets. Fecal LA was positively correlated } \\
\text { with fasting LDL-C, while total SBA, LA and DCA were negatively } \\
\text { correlated with fasting HDL-C. }\end{array}$ & Favorable \\
\hline \multirow[t]{6}{*}{$\begin{array}{l}\text { Dietary } \\
\text { Protein }\end{array}$} & $\begin{array}{l}\text { Rodent (mice and rats) } \\
{[65]}\end{array}$ & $\begin{array}{l}\text { HP diet rich in either beans, vegetables, dairy, seafood, or meat had } \\
\text { differential effects on circulating BA concentrations as well as BA profiles. }\end{array}$ & $\begin{array}{l}\text { Mixed depending } \\
\text { on protein source }\end{array}$ \\
\hline & $\begin{array}{l}\text { Human } \\
{[66]}\end{array}$ & $\begin{array}{l}\text { VLCD high in protein decreased fecal total BA concentration and reduced } \\
\text { deconjugation and 7-a-dihydroxylation, accompanied by significant } \\
\text { changes in several bacterial taxa. Individual fecal BA correlated with } \\
\text { amino acid, purine, and lipid metabolic pathways in plasma and adipose } \\
\text { tissue transcriptome. }\end{array}$ & Favorable \\
\hline & $\begin{array}{l}\text { Rodent (rat) } \\
{[67]}\end{array}$ & $\begin{array}{l}\text { SPI versus casein diet increased BA synthesis via FGF15 and SHP, and } \\
\text { accelerated BA excretion. }\end{array}$ & Favorable \\
\hline & $\begin{array}{l}\text { Rodent (C57BL/6 mice) } \\
\text { [71] }\end{array}$ & $\begin{array}{l}\text { HFD+SPI compared to HFD+dairy protein increased BA pool with higher } \\
\text { SBA/PBA ratio. No change in BA excretion. Increased secretion of } \\
\text { intestinal GLP-1. Expansion of taxa involved in BA biotransformation. }\end{array}$ & Favorable \\
\hline & $\begin{array}{l}\text { Rodent (rats) } \\
\text { [72] }\end{array}$ & $\begin{array}{l}\text { Dietary pea protein versus casein increased BA synthesis and BA } \\
\text { excretion resulting in reduced hepatic cholesterol content and secretion of } \\
\text { VLDL-C. Compensatory increase in gene expression of SREBP-2, HMG- } \\
\text { CoA reductase and LDL receptor. }\end{array}$ & Favorable \\
\hline & $\begin{array}{l}\text { Human } \\
{[68]}\end{array}$ & $\begin{array}{l}\text { HFHP compared to a HF diet decreased intrahepatocellular lipids and } \\
\text { increased plasma CA, CDCA and DCA levels, suggesting that HP blunts } \\
\text { effect of HF diets. }\end{array}$ & Favorable \\
\hline
\end{tabular}




\begin{tabular}{|c|c|c|c|}
\hline & Human[73] & $\begin{array}{l}\text { HP (milk protein) increased TBA and PBA concentrations. The MIX diet } \\
\text { (both cereal fiber and protein) increased circulating BA concentrations in } \\
\text { obese participants, suggesting a compensatory increase of the BA pool or } \\
\text { defective transport, in this sub-group. }\end{array}$ & $\begin{array}{l}\text { Mixed depending } \\
\text { on protein source. } \\
\text { Unfavorable in } \\
\text { obese subgroup. }\end{array}$ \\
\hline \multirow[t]{4}{*}{$\begin{array}{r}\text { Dietary } \\
\text { Carbohydrate }\end{array}$} & Porcine[76] & $\begin{array}{l}\text { Oat } \beta \text {-glucan altered BA profile, decreased circulating TBA, TC and LDL-C } \\
\text { concentrations and reduced reabsorption of BA across the intestinal } \\
\text { epithelium. }\end{array}$ & Favorable \\
\hline & Human[77] & $\begin{array}{l}\text { Whole grain compared to refined grain diet increased TLCA, TCA and } \\
\text { GCA concentrations. Plasma TBA, specifically } 12 \alpha \text {-hydroxylated BAs were } \\
\text { positively associated with insulin resistance, potentially mediated by FXR } \\
\text { and TGR5. }\end{array}$ & Favorable \\
\hline & $\begin{array}{l}\text { Humans } \\
{[80]}\end{array}$ & $\begin{array}{l}\text { Isocaloric high carbohydrate versus lipid beverage decreased circulating } \\
\text { BA concentrations, but no association with FGF- } 19 \text { levels. }\end{array}$ & Unclear \\
\hline & $\begin{array}{l}\text { Rodent (rat) } \\
{[81]}\end{array}$ & $\begin{array}{l}\text { High sucrose versus complex carbohydrate diet decreased serum 7-alpha- } \\
\text { hydroxy-4-cholesten-3-one and hepatic Cyp7a1 mRNA expression, } \\
\text { suggesting decreased BA synthesis. }\end{array}$ & Unclear \\
\hline \multicolumn{4}{|c|}{. } \\
\hline Polyphenols & $\begin{array}{l}\text { In vitro and Rodent } \\
\text { [82] }\end{array}$ & $\begin{array}{l}\text { Flavonoids, resveratrol, phenolics, and quercetin have been shown to } \\
\text { increase BA excretion with corresponding reductions in TC and LDL-C } \\
\text { concentrations potentially linked to changes in gut microbiota composition, } \\
\text { reduced expression of intestinal BA transporters and/or an increase in } \\
\text { CYP7A1 activity and LXRa signaling. }\end{array}$ & Favorable \\
\hline Cranberries & $\begin{array}{l}\text { Human } \\
{[83]}\end{array}$ & $\begin{array}{l}\text { Animal protein-based diet with freeze dried cranberry powder decreased } \\
\text { abundance of Firmicutes and increased abundance of Bacteroidetes, } \\
\text { compared to placebo powder. The cranberry diet attenuated animal-based } \\
\text { diet induced increase in fecal SBA. }\end{array}$ & Favorable \\
\hline \multirow[t]{2}{*}{ Walnuts } & $\begin{array}{l}\text { Human } \\
{[84]}\end{array}$ & $\begin{array}{l}\text { Compared to a control diet, walnut intake increased relative abundances } \\
\text { of Firmicutes species in butyrate-producing Clostridium clusters XIVa and } \\
\text { IV, including Faecalibacterium and Roseburia. Decreased fecal SBA and } \\
\text { plasma LDL-C concentrations. }\end{array}$ & Favorable \\
\hline & $\begin{array}{l}\text { Human } \\
{[85]}\end{array}$ & $\begin{array}{l}\text { Whole walnuts or ALA-matched diet resulted in enrichment of bacteria } \\
\text { associated with SBA production, and lowered plasma TC, nHDL-C and } \\
\text { LDL- concentrations, relative to a diet matched for SFA but with oleic acid } \\
\text { in place of ALA. }\end{array}$ & Favorable \\
\hline $\begin{array}{l}\text { L-carnitine } \\
\text { (red meat) }\end{array}$ & $\begin{array}{l}\text { Rodent (apoE-l- mice) } \\
\text { and Human } \\
\text { [87] }\end{array}$ & $\begin{array}{l}\text { Dietary L-carnitine supplementation or D3-L-carnitne increased production } \\
\text { of the pro-atherosclerotic metabolite TMAO, which decreased the BA pool } \\
\text { size and reduced key BA synthesis and transport proteins. This was } \\
\text { associated with atherosclerosis development in mice and increased CVD } \\
\text { risk in humans. }\end{array}$ & Unfavorable \\
\hline Probiotics & Rodent (mice) & VSL\#3 (containing BSH active probiotic strains) increased fecal BA & Favorable \\
\hline
\end{tabular}




\begin{tabular}{|l|l|l|l|}
\hline & {$[92]$} & deconjugation and excretion, as well as hepatic BA synthesis. & \\
\hline $\begin{array}{l}\text { Dietary } \\
\text { Patterns }\end{array}$ & Human & Western-type diet resulted in higher colonic concentrations of SBA and & Unfavorable \\
& was associated with higher CVD risk. & $\begin{array}{l}\text { Vegan/vegetarian and Mediterranean diets resulted in lower fecal SBA } \\
\text { concentrations and were associated with lower CVD risk. }\end{array}$ & Favorable \\
\cline { 2 - 5 } & $\begin{array}{l}\text { Human } \\
{[70,93,94]}\end{array}$ & \\
\hline
\end{tabular}

${ }^{1}$ Abbreviations: CA: cholic acid; CDCA: chenodeoxycholic acid; CVD: cardiovascular disease; DCA; deoxycholic acid; FGF: fibroblast growth factor; GCA: glycocholic acid; GLP-1: glucagon like peptide-1; HCF: high cereal fiber; HDL-C: highdensity lipoprotein cholesterol; HF: high fat; HFD: high fat diet; HMG-CoA: 3-hydroxy-3-methyl-glutaryl-coenzyme A; HP: high protein; LA: lithocholic acid; LDL-C: low-density lipoprotein cholesterol; LF: low fat; PBA: primary bile acid; PUFA: polyunsatured fatty acid; SBA: secondary bile acid; SFA: saturated fatty acid; SHP: small heterodimer partner; SPI: soy protein isolate; SREBP-2: sterol regulating element binding protein-2; TCA: taurocholic acid; VLCD: very low calorie diet; VLDL-C: very-low density lipoprotein cholesterol. 


\section{REFERENCES}

1. Sonnenburg JL, Bäckhed F. Diet-microbiota interactions as moderators of human metabolism. Nature 2016: 535(7610):56-64.

2. David LA, Maurice CF, Carmody RN, et al. Diet rapidly and reproducibly alters the human gut microbiome. Nature 2014: 505(7484):559-563.

3. Sheflin AM, Melby CL, Carbonero F, Weir TL. Linking dietary patterns with gut microbial composition and function. Gut Microbes 2017: 8(2):113-129.

4. Jonsson AL, Bäckhed F. Role of gut microbiota in atherosclerosis. Nat Rev Cardiol 2017: 14(2):79-87.

5. Wang Z, Zhao Y. Gut microbiota derived metabolites in cardiovascular health and disease. Protein Cell 2018: 9(5):416-431.

\section{- Detailed overview of various gut-derived metabolites and their role in CVD.}

6. Martinez KB, Leone V, Chang EB. Microbial metabolites in health and disease: Navigating the unknown in search of function. J Biol Chem 2017: 292(21):85538559.

7. Tang WHW, Bäckhed F, Landmesser U, Hazen SL. Intestinal Microbiota in Cardiovascular Health and Disease (JACC State-of-the-Art Review). J Am Coll Cardiol 2019: 73(16):2089-2105.

- Recent review focused on human intestinal microbiota and its potential modulation to improve cardiovascular health.

8. Fu BC, Hullar MAJ, Randolph TW, et al. Associations of plasma trimethylamine Noxide, choline, carnitine, and betaine with inflammatory and cardiometabolic risk biomarkers and the fecal microbiome in the Multiethnic Cohort Adiposity Phenotype Study. Am J Clin Nutr 2020: 13(10).

9. Wahlström A, Sayin SI, Marschall HU, Bäckhed F. Intestinal Crosstalk between Bile Acids and Microbiota and Its Impact on Host Metabolism. Cell Metab 2016: 24(1):41-50.

10. McMillin M, DeMorrow S. Effects of bile acids on neurological function and disease. Faseb J 2016: 30(11):3658-3668.

11. Feng Q, Liu Z, Zhong S, et al. Integrated metabolomics and metagenomics analysis of plasma and urine identified microbial metabolites associated with coronary heart disease. Sci Rep 2016: 6(22525). 
12. Lin CJ, Chuang CK, Jayakumar T, et al. Serum p-cresyl sulfate predicts cardiovascular disease and mortality in elderly hemodialysis patients. Arch Med Sci 2013: 9(4):662-668.

13. Poesen R, Claes K, Evenepoel P, et al. Microbiota-Derived Phenylacetylglutamine Associates with Overall Mortality and Cardiovascular Disease in Patients with CKD. J Am Soc Nephrol 2016: 27(11):3479-3487.

14. Hofmann AF. The continuing importance of bile acids in liver and intestinal disease. Arch Int Med 1999: 159(22):2647-2658.

15. Šarenac TM, Mikov M. Bile Acid Synthesis: From Nature to the Chemical Modification and Synthesis and Their Applications as Drugs and Nutrients. Front Pharmacol 2018: 9(939).

16. Fiorucci S, Distrutti E. Bile Acid-Activated Receptors, Intestinal Microbiota, and the Treatment of Metabolic Disorders. Trends Molec Med 2015: 21(11):702-714.

17. Alnouti Y. Bile Acid Sulfation: A Pathway of Bile Acid Elimination and Detoxification. Toxicological Sciences 2009: 108(2):225-246.

18. Hofmann AF, Hagey LR. Bile acids: chemistry, pathochemistry, biology, pathobiology, and therapeutics. Cell Mol Life Sci 2008: 65(16):2461-2483.

19. Di Ciaula A, Garruti G, Lunardi Baccetto R, et al. Bile Acid Physiology. Ann Hepatol 2017: 16(Suppl. 1: s3-105.):s4-s14.

20. Russell DW. The enzymes, regulation, and genetics of bile acid synthesis. Ann Rev Biochem 2003: 72:137-174.

21. Sinal CJ, Tohkin M, Miyata M, Ward JM, Lambert G, Gonzalez FJ. Targeted disruption of the nuclear receptor FXR/BAR impairs bile acid and lipid homeostasis. Cell 2000: 102(6):731-744.

22. Norlin M, Wikvall K. Enzymes in the conversion of cholesterol into bile acids. Curr Mol Med 2007: 7(2):199-218.

23. Hundt M, Basit H, John S. Physiology, Bile Secretion. 2020.

24. Molinero N, Ruiz L, Sánchez B, Margolles A, Delgado S. Intestinal Bacteria Interplay With Bile and Cholesterol Metabolism: Implications on Host Physiology. Front Physiol 2019: 10:185-185.

- A detailed overview of bile acid physiology and its reciprocal relationship with gut microbiota.

25. Chiang JYL. Bile acids: regulation of synthesis. J Lip Res 2009: 50(10):1955-1966. 
26. Busnelli M, Manzini S, Chiesa G. The Gut Microbiota Affects Host Pathophysiology as an Endocrine Organ: A Focus on Cardiovascular Disease. Nutrients 2019: 12(1).

27. Chen ML, Takeda K, Sundrud MS. Emerging roles of bile acids in mucosal immunity and inflammation. Mucosal Immunol 2019: 12(4):851-861.

28. Sarafian MH, Lewis MR, Pechlivanis A, et al. Bile acid profiling and quantification in biofluids using ultra-performance liquid chromatography tandem mass spectrometry. Anal Chem 2015: 87(19):9662-9670.

29. Kliewer SA, Mangelsdorf DJ. Bile Acids as Hormones: The FXR-FGF15/19 Pathway. Dig Dis 2015: 33(3):327-331.

30. Wang C, Zhu C, Shao L, Ye J, Shen Y, Ren Y. Role of Bile Acids in Dysbiosis and Treatment of Nonalcoholic Fatty Liver Disease. Mediators Inflamm 2019: 2019:7659509-7659509.

31. Schaap FG, Trauner M, Jansen PLM. Bile acid receptors as targets for drug development. Nat Rev Gastroenterol Hepatol 2014: 11(1):55-67.

32. Makishima M, Okamoto AY, Repa JJ, et al. Identification of a nuclear receptor for bile acids. Science 1999: 284(5418):1362-1365.

33. Song P, Rockwell CE, Cui JY, Klaassen CD. Individual bile acids have differential effects on bile acid signaling in mice. Toxicol Appl Pharmacol 2015: 283(1):57-64.

34. Khurana S, Raufman J-P, Pallone TL. Bile acids regulate cardiovascular function. Clin Trans Sci 2011: 4(3):210-218.

35. Sannasiddappa TH, Lund PA, Clarke SR. In Vitro Antibacterial Activity of Unconjugated and Conjugated Bile Salts on Staphylococcus aureus. Front Microbiol 2017: 8:1581-1581.

36. Jia W, Xie G, Jia W. Bile acid-microbiota crosstalk in gastrointestinal inflammation and carcinogenesis. Nat Rev Gastroenterol Hepatol 2018: 15(2):111-128.

37. Trottier J, Caron P, Straka RJ, Barbier O. Profile of serum bile acids in noncholestatic volunteers: gender-related differences in response to fenofibrate. Clin Pharmacol Ther 2011: 90(2):279-286.

38. Charach G, Karniel E, Novikov I, et al. Reduced bile acid excretion is an independent risk factor for stroke and mortality: A prospective follow-up study. Atherosclerosis 2020: 293:79-85.

39. Li Y, Zhang D, He Y, et al. Investigation of novel metabolites potentially involved in the pathogenesis of coronary heart disease using a UHPLC-QTOF/MS-based metabolomics approach. Sci Rep 2017: 7(1):017-15737. 
40. Charach G, Grosskopf I, Rabinovich A, Shochat M, Weintraub M, Rabinovich P. The association of bile acid excretion and atherosclerotic coronary artery disease. Ther Adv Gastroenterol 2011: 4(2):95-101.

41. Rajaratnam RA, Gylling H, Miettinen TA. Cholesterol absorption, synthesis, and fecal output in postmenopausal women with and without coronary artery disease. Arterioscler Thromb Vasc Biol 2001: 21(10):1650-1655.

42. Li W, Shu S, Cheng L, et al. Fasting serum total bile acid level is associated with coronary artery disease, myocardial infarction and severity of coronary lesions. Atherosclerosis 2020: 292:193-200.

43. Patti ME, Houten SM, Bianco AC, et al. Serum bile acids are higher in humans with prior gastric bypass: potential contribution to improved glucose and lipid metabolism. Obesity 2009: 17(9):1671-1677.

44. Sun W, Zhang D, Wang Z, et al. Insulin Resistance is Associated With Total Bile Acid Level in Type 2 Diabetic and Nondiabetic Population: A Cross-Sectional Study. Medicine 2016: 95(10):0000000000002778.

45. Battson ML, Lee DM, Weir TL, Gentile CL. The gut microbiota as a novel regulator of cardiovascular function and disease. J Nutr Biochem 2018: 56:1-15.

46. Vasavan T, Ferraro E, Ibrahim E, Dixon P, Gorelik J, Williamson C. Heart and bile acids - Clinical consequences of altered bile acid metabolism. Biochim Biophys Acta Mol Basis Dis 2018: 1864(4 Pt B):1345-1355.

47. Zhang $\mathrm{Y}$, Wang $\mathrm{X}$, Vales $\mathrm{C}$, et al. FXR deficiency causes reduced atherosclerosis in Ldlr-/- mice. Arterioscler Thromb Vasc Biol 2006: 26(10):2316-2321.

48. Pols TW, Nomura M, Harach $\mathrm{T}$, et al. TGR5 activation inhibits atherosclerosis by reducing macrophage inflammation and lipid loading. Cell Metab 2011: 14(6):747757.

49. Jadhav K, Xu Y, Li Y, et al. Reversal of metabolic disorders by pharmacological activation of bile acid receptors TGR5 and FXR. Mol Metab 2018: 9:131-140.

50. Miyazaki-Anzai S, Masuda M, Kohno S, et al. Simultaneous inhibition of FXR and TGR5 exacerbates atherosclerotic formation. J Lip Res 2018: 59(9):1709-1713.

51. Ma J, Li H. The Role of Gut Microbiota in Atherosclerosis and Hypertension. Front Pharmacol 2018: 9:1082-1082.

52. Kundu S, Bansal S, Muthukumarasamy KM, Sachidanandan C, Motiani RK, Bajaj A. Deciphering the role of hydrophobic and hydrophilic bile acids in angiogenesis using in vitro and in vivo model systems. Medchemcomm 2017: 8(12):2248-2257.

53. Takahashi S, Luo Y, Ranjit S, et al. Bile Acid Sequestration Reverses Liver Injury and Prevents Progression of NASH in Western Diet-Fed Mice. J Biol Chem 2020:jbc.RA119.011913. 
54. Hofmann AF. Pharmacology of ursodeoxycholic acid, an enterohepatic drug. Scand J Gastroenterol Suppl 1994: 204:1-15.

55. Abenavoli L, Falalyeyeva T, Boccuto L, Tsyryuk O, Kobyliak N. Obeticholic Acid: A New Era in the Treatment of Nonalcoholic Fatty Liver Disease. Pharmaceuticals 2018: 11(4):104.

56. Đanić M, Stanimirov B, Pavlović N, et al. Pharmacological Applications of Bile Acids and Their Derivatives in the Treatment of Metabolic Syndrome. Front Pharmacol 2018: 9(1382).

57. Singh RK, Chang HW, Yan D, et al. Influence of diet on the gut microbiome and implications for human health. J Transl Med 2017: 15(1):017-1175.

58. Kasubuchi M, Hasegawa S, Hiramatsu T, Ichimura A, Kimura I. Dietary gut microbial metabolites, short-chain fatty acids, and host metabolic regulation. Nutrients 2015: 7(4):2839-2849.

59. Yokota A, Fukiya S, Islam KB, et al. Is bile acid a determinant of the gut microbiota on a high-fat diet? Gut Microbes 2012: 3(5):455-459.

60. Bisschop PH, Bandsma RH, Stellaard F, et al. Low-fat, high-carbohydrate and high-fat, low-carbohydrate diets decrease primary bile acid synthesis in humans. Am J Clin Nutr 2004: 79(4):570-576.

61. Brassard D, Tessier-Grenier M, Allaire J, et al. Comparison of the impact of SFAs from cheese and butter on cardiometabolic risk factors: a randomized controlled trial. Am J Clin Nutr 2017: 105(4):800-809.

62. Mokkala K, Houttu N, Cansev T, Laitinen K. Interactions of dietary fat with the gut microbiota: Evaluation of mechanisms and metabolic consequences. Clin Nutr 2020: 39(4):994-1018.

63. Devkota S, Chang EB. Interactions between Diet, Bile Acid Metabolism, Gut Microbiota, and Inflammatory Bowel Diseases. Dig Dis 2015: 33(3):351-356.

64. Meng $\mathrm{H}$, Matthan NR, Wu D, et al. Comparison of diets enriched in stearic, oleic, and palmitic acids on inflammation, immune response, cardiometabolic risk factors, and fecal bile acid concentrations in mildly hypercholesterolemic postmenopausal women-randomized crossover trial. Am J Clin Nutr 2019: 110(2):305-315.

65. Madsen L, Myrmel LS, Fjære E, Liaset B, Kristiansen K. Links between Dietary Protein Sources, the Gut Microbiota, and Obesity. Front Physiol 2017: 8(1047).

66. Alemán JO, Bokulich NA, Swann JR, et al. Fecal microbiota and bile acid interactions with systemic and adipose tissue metabolism in diet-induced weight loss of obese postmenopausal women. J Transl Med 2018: 16(1):018-1619. 
67. Arellano-Martínez GL, Granados O, Palacios-González B, Torres N, Medina-Vera I, Tovar AR. Soya protein stimulates bile acid excretion by the liver and intestine through direct and indirect pathways influenced by the presence of dietary cholesterol. Br J Nutr 2014: 111(12):2059-2066.

68. Bortolotti M, Kreis R, Debard C, et al. High protein intake reduces intrahepatocellular lipid deposition in humans. Am J Clin Nutr 2009: 90(4):10021010.

69. Tomova A, Bukovsky I, Rembert E, et al. The Effects of Vegetarian and Vegan Diets on Gut Microbiota. Front Nutr 2019: 6(47).

70. Trefflich I, Marschall HU, Giuseppe RD, et al. Associations between Dietary Patterns and Bile Acids-Results from a Cross-Sectional Study in Vegans and Omnivores. Nutrients 2019: 12(1).

71. Watanabe K, Igarashi M, Li X, et al. Dietary soybean protein ameliorates high-fat diet-induced obesity by modifying the gut microbiota-dependent biotransformation of bile acids. PLOS ONE 2018: 13(8).

72. Spielmann J, Stangl GI, Eder K. Dietary pea protein stimulates bile acid excretion and lowers hepatic cholesterol concentration in rats. J Anim Physiol Anim Nutr 2008: 92(6):683-693.

73. Weickert MO, Hattersley JG, Kyrou I, et al. Effects of supplemented isoenergetic diets varying in cereal fiber and protein content on the bile acid metabolic signature and relation to insulin resistance. Nutr Diabetes 2018: 8(1):018-0020.

74. Hills RD, Jr., Pontefract BA, Mishcon HR, Black CA, Sutton SC, Theberge CR. Gut Microbiome: Profound Implications for Diet and Disease. Nutrients 2019: 11(7).

75. Soliman GA. Dietary Fiber, Atherosclerosis, and Cardiovascular Disease. Nutrients 2019: 11(5).

76. Gunness P, Michiels J, Vanhaecke L, et al. Reduction in circulating bile acid and restricted diffusion across the intestinal epithelium are associated with a decrease in blood cholesterol in the presence of oat $\beta$-glucan. Faseb J 2016: 30(12):42274238.

77. Ginos BNR, Navarro SL, Schwarz Y, et al. Circulating bile acids in healthy adults respond differently to a dietary pattern characterized by whole grains, legumes and fruits and vegetables compared to a diet high in refined grains and added sugars: A randomized, controlled, crossover feeding study. Metabolism 2018: 83:197-204.

78. Connolly ML, Tzounis X, Tuohy KM, Lovegrove JA. Hypocholesterolemic and Prebiotic Effects of a Whole-Grain Oat-Based Granola Breakfast Cereal in a Cardio-Metabolic "At Risk" Population. Front Microbiol 2016: 7(1675). 
79. Hollænder PL, Ross AB, Kristensen M. Whole-grain and blood lipid changes in apparently healthy adults: a systematic review and meta-analysis of randomized controlled studies. Am J Clin Nutr 2015: 102(3):556-572.

80. Morton GJ, Kaiyala KJ, Foster-Schubert KE, Cummings DE, Schwartz MW. Carbohydrate feeding dissociates the postprandial FGF19 response from circulating bile acid levels in humans. J Clin Endocrinol Metab 2014: 99(2):20133129.

81. Apro J, Beckman L, Angelin B, Rudling M. Influence of dietary sugar on cholesterol and bile acid metabolism in the rat: Marked reduction of hepatic Abcg5/8 expression following sucrose ingestion. Biochem Biophys Res Comm 2015: $461(4): 592-597$.

82. Chambers KF, Day PE, Aboufarrag HT, Kroon PA. Polyphenol Effects on Cholesterol Metabolism via Bile Acid Biosynthesis, CYP7A1: A Review. Nutrients 2019: 11(11).

83. Rodríguez-Morató J, Matthan NR, Liu J, de la Torre R, Chen CO. Cranberries attenuate animal-based diet-induced changes in microbiota composition and functionality: a randomized crossover controlled feeding trial. J Nutr Biochem 2018: 62:76-86.

84. Holscher HD, Guetterman HM, Swanson KS, et al. Walnut Consumption Alters the Gastrointestinal Microbiota, Microbially Derived Secondary Bile Acids, and Health Markers in Healthy Adults: A Randomized Controlled Trial. J Nutr 2018: 148(6):861-867.

85. Tindall AM, McLimans CJ, Petersen KS, Kris-Etherton PM, Lamendella R. Walnuts and Vegetable Oils Containing Oleic Acid Differentially Affect the Gut Microbiota and Associations with Cardiovascular Risk Factors: Follow-up of a Randomized, Controlled, Feeding Trial in Adults at Risk for Cardiovascular Disease. J Nutr 2020: 150(4):806-817.

86. Koeth RA, Wang Z, Levison BS, et al. Intestinal microbiota metabolism of Lcarnitine, a nutrient in red meat, promotes atherosclerosis. Nat Med 2013: 19(5):576-585.

87. Koeth RA, Lam-Galvez BR, Kirsop J, et al. L-Carnitine in omnivorous diets induces an atherogenic gut microbial pathway in humans. J Clin Invest 2019: 129(1):373387.

88. Ding L, Chang M, Guo Y, et al. Trimethylamine-N-oxide (TMAO)-induced atherosclerosis is associated with bile acid metabolism. Lipids Health Dis 2018: 17(1):018-0939.

89. Pavlović N, Stankov K, Mikov M. Probiotics--interactions with bile acids and impact on cholesterol metabolism. Appl Biochem Biotechnol 2012: 168(7):1880-1895. 
90. Jones ML, Tomaro-Duchesneau C, Prakash S. The gut microbiome, probiotics, bile acids axis, and human health. Trends Microbiol 2014: 22(6):306-308.

91. Joyce SA, MacSharry J, Casey PG, et al. Regulation of host weight gain and lipid metabolism by bacterial bile acid modification in the gut. PNAS 2014: 111(20):7421-7426.

92. Degirolamo C, Rainaldi S, Bovenga F, Murzilli S, Moschetta A. Microbiota modification with probiotics induces hepatic bile acid synthesis via downregulation of the Fxr-Fgf15 axis in mice. Cell Rep 2014: 7(1):12-18.

93. Tindall AM, Petersen KS, Kris-Etherton PM. Dietary Patterns Affect the Gut Microbiome-The Link to Risk of Cardiometabolic Diseases. J Nutr 2018: 148(9):1402-1407.

\section{- Brief review of the relationship between specific dietary patterns and cardiovascular disease.}

94. Bailey MA, Holscher HD. Microbiome-Mediated Effects of the Mediterranean Diet on Inflammation. Adv Nutr 2018: 9(3):193-206.

95. Sheng L, Jena PK, Liu HX, et al. Gender Differences in Bile Acids and Microbiota in Relationship with Gender Dissimilarity in Steatosis Induced by Diet and FXR Inactivation. Sci Rep 2017: 7(1):017-01576.

96. Baars $\mathrm{A}$, Oosting $\mathrm{A}$, Lohuis $\mathrm{M}$, et al. Sex differences in lipid metabolism are affected by presence of the gut microbiota. Sci Rep 2018: 8(1):018-31695.

97. Kaddurah-Daouk R, Baillie RA, Zhu H, et al. Enteric microbiome metabolites correlate with response to simvastatin treatment. PLOS ONE 2011: 6(10):e25482e25482.

98. Miyata M, Takamatsu $\mathrm{Y}$, Kuribayashi $\mathrm{H}$, Yamazoe $\mathrm{Y}$. Administration of ampicillin elevates hepatic primary bile acid synthesis through suppression of ileal fibroblast growth factor 15 expression. J Pharmacol Exp Ther 2009: 331(3):1079-1085.

99. Wang W, Cheng Z, Wang Y, Dai Y, Zhang X, Hu S. Role of Bile Acids in Bariatric Surgery. Front Physiol 2019: 10(374).

100. Steiner C, Othman A, Saely $\mathrm{CH}$, et al. Bile acid metabolites in serum: intraindividual variation and associations with coronary heart disease, metabolic syndrome and diabetes mellitus. PLOS ONE 2011: 6(11):14.

101. Yin S, Su M, Xie G, et al. Factors affecting separation and detection of bile acids by liquid chromatography coupled with mass spectrometry in negative mode. Anal Bioanal Chem 2017: 409(23):5533-5545. 
102. Thakare R, Alamoudi JA, Gautam N, Rodrigues AD, Alnouti Y. Species differences in bile acids I. Plasma and urine bile acid composition. J Appl Toxicol 2018: 38(10):1323-1335.

103. Rodríguez-Morató J, Pozo Ó J, Marcos J. Targeting human urinary metabolome by LC-MS/MS: a review. Bioanalysis 2018: 10(7):489-516.

104. Steiner C, von Eckardstein A, Rentsch KM. Quantification of the 15 major human bile acids and their precursor 7a-hydroxy-4-cholesten-3-one in serum by liquid chromatography-tandem mass spectrometry. J Chromatogr B 2010: 878(28):28702880 .

105. Reinicke M, Schröter J, Müller-Klieser D, Helmschrodt C, Ceglarek U. Free oxysterols and bile acids including conjugates - Simultaneous quantification in human plasma and cerebrospinal fluid by liquid chromatography-tandem mass spectrometry. Anal Chim Acta 2018: 11:245-255.

106. Dutta M, Cai J, Gui W, Patterson AD. A review of analytical platforms for accurate bile acid measurement. Anal Bioanal Chem 2019: 411(19):4541-4549.

- Recent summary of different detection technologies commonly employed for the measurement of bile acids

107. Hosomi R, Matsudo A, Sugimoto K, et al. Dietary Fat Influences the Expression of Genes Related to Sterol Metabolism and the Composition of Cecal Microbiota and Its Metabolites in Rats. J Oleo Sci 2019: 68(11):1133-1147. 